\title{
CYCLES ALGÉBRIQUES ET HOMOMORPHISMES EN HOMOLOGIE D'INTERSECTION
}

\author{
By JEAN - PAUL BRASSELET
}

Les trois chapitres qui suivent ont pour but d'introduire les résultats récents dûs à Gottfried Barthel, Karl-Heinz Fieseler, Ofer Gabber, Ludger Kaup et l'auteur ([2]).

\section{Premier chapitre}

1. Faisceau des chaînes singulières. ([3; $\mathrm{I}, 1,2],[5])$

DÉfinition. On dit qu'un espace topologique $X$ est muni d'une structure PL si on s'est donné une classe de triangulations localement finies de $X$ (triangulations admissibles) satisfaisant :

1) toute subdivision (barycentrique ou linéaire) d'une triangulation admissible est admissible.

2) Deux triangulations admissibles $K_{1}$ et $K_{2}$ admettent une subdivision (admissible) commune.

Tout ouvert $U$ de $X$ admet une structure PL induite : Pour toutes triangulations admissibles $K$ de $X$ et $K_{U}$ de $U$, il existe une subdivision linéaire $K_{U}^{\prime}$ de $K_{U}$ telle que tout simplexe de $K_{U}^{\prime}$ est contenu (linéairement) dans un simplexe de $K$.

Chaînes et supports.

Soit $K$ une triangulation de $X$, on note $C_{i}^{F}(K)$ le groupe des $i$-chaînes à supports fermés, i.e. les combinaisons linéaires localement finies à coefficients rationnels $\xi=\sum \xi_{\sigma} \sigma$ (chaînes de deuxième espèce chez Cartan). Le support de la chaîne $\xi \in C_{i}^{F}(K)$, est $|\xi|=\bigcup_{\xi_{\sigma} \neq 0}|\sigma|$. C'est un fermé de $X$. De même, $C_{i}(K)$ désigne le groupe des $i$-chaînes à supports compacts, i.e. les combinaisons linéaires $\xi=\sum \xi_{\sigma} \sigma$ pour lesquelles tous les $\xi_{\sigma}$ sont nuls sauf un nombre fini.

Le groupe des $i$-chaînes PL à supports fermés de $X$, noté $C_{i}^{F}(X)$, est défini comme la limite directe des $C_{i}^{F}(K)$ pour toutes les triangulations de $X$, c'est-à-dire la réunion des groupes $C_{i}^{F}(K)$ pour toutes les triangulations admissibles $K$ modulo l'identification de deux chaînes $\xi \in C_{i}^{F}(K)$ et $\xi^{\prime} \in C_{i}^{F}\left(K^{\prime}\right)$ s'il existe une subdivision commune $K^{\prime \prime}$ de $K$ et $K^{\prime}$ telle que les images canoniques de $\xi$ et $\xi^{\prime}$ dans $C_{i}^{F}\left(K^{\prime \prime}\right)$ coïncident.

On définit de même $C_{i}(X)$.

Les groupes d'homologie de $X$ à support fermé sont les groupes d'homologie du complexe $C_{*}^{F}(X)$, on les note $H_{\imath}^{F}(X)$. Les groupes d'homologie à support compact sont les groupes d'homologie du complexe $C_{*}(X)$, on les note $H_{i}(X)$. 
Si $U$ est ouvert dans $X$, l'inclusion de $U$ dans $X$ induit des morphismes

$$
\rho_{U X}: C_{i}^{F}(X) \rightarrow C_{i}^{F}(U)
$$

de la façon suivante : Soit $\xi \in C_{i}^{F}(X)$, il existe une triangulation $K$ de $X$ telle que $\xi$ s'écrit $\sum_{\sigma \in K} \xi_{\sigma} \sigma$. Etant donnée une triangulation de $U$, elle admet une subdivision $K_{U}$ telle que tout simplexe $\tau$ de $K_{U}$ soit contenu dans un simplexe de même dimension $\sigma(\tau)$ d'une subdivision de $K$. A la chaîne $\xi$, on fait correspondre la chaîne :

$$
\rho_{U X}(\xi)=\sum_{\tau \in K_{U}}[\tau: \sigma(\tau)] \xi_{\sigma(\tau)} \tau
$$

où

$$
[\tau: \sigma]= \begin{cases}0 & \text { si } \tau \not \subset \sigma \\ +1 & \text { si } \tau \subset \sigma \text { avec même orientation } \\ -1 & \text { si } \tau \subset \sigma \text { avec orientation opposée. }\end{cases}
$$

Les morphismes (1) commutent au bord et induisent des morphismes en homologie :

$$
H_{\imath}^{F}(X) \rightarrow H_{\imath}^{F}(U)
$$

Si $U \subset V$ sont deux ouverts de $X$, on définit comme en (1) une application naturelle $\rho_{U V}: C_{i}^{F}(V) \rightarrow C_{i}^{F}(U)$. Si $\xi \in C_{i}^{F}(V)$ s'écrit $\xi=\sum_{\sigma \in K_{V}} \xi_{\sigma} \sigma$ pour une triangulation localement finie $K_{V}$ de $V$, il existe comme précédemment une triangulation $K_{U}$ de $U$ telle que tout simplexe $\tau$ de $K_{U}$ soit contenu dans un simplexe $\sigma(\tau)$ d'une subdivision de $K_{V}$. A la chaîne $\xi$ on fait correspondre la chaîne $\rho_{V U}(\xi) \in C_{i}^{F}(U)$ définie par

$$
\rho_{U V}(\xi)=\sum_{\tau \in K_{U}}[\tau: \sigma(\tau)] \xi_{\sigma(\tau)} \tau
$$

et $\left|\rho_{U V}(\xi)\right|=|\xi| \cap U$. La correspondance $U \mapsto C_{i}^{F}(U)$ définit un préfaisceau, qui est un faisceau. Si $\operatorname{dim} X=2 m$, nous notons ce faisceau $\mathcal{C}^{\bullet}=\mathcal{C}_{2 m-\bullet}^{F}$ et plus précisément $\mathcal{C}_{X}^{\bullet}$ lorsqu'il sera nécessaire de préciser l'espace $X$. On a $\mathcal{C}^{\bullet}(U)=\mathcal{C}_{2 m-\bullet}^{F}(U)$.

Lemme. Les faisceaux $\mathcal{C}^{q}$ sont fins $([6],[8 ; \mathrm{II}, 3.7])$. On a donc, pour tout $q$ :

$$
H^{p}\left(X ; \mathcal{C}_{X}^{q}\right)=0 \quad \forall p>0
$$

2. Hypercohomologie. ([3; II.5; V,1.4] et [8; II.5.2-3])

Etant donné un complexe de faisceaux $\mathcal{A}^{\bullet}$ sur $X$, et $\mathcal{U}$ un recouvrement ouvert localement fini de $X$, on note $C^{p}\left(\mathcal{U} ; \mathcal{A}^{q}\right)$ l'ensemble des $p$-cochaînes de Čech sur $\mathcal{U}$ à coefficients dans $\mathcal{A}^{q}$,

$$
\delta_{1}: C^{p}\left(\mathcal{U} ; \mathcal{A}^{q}\right) \rightarrow C^{p+1}\left(\mathcal{U} ; \mathcal{A}^{q}\right)
$$

la différentielle induite par celle de Čech et

$$
\delta_{2}: C^{p}\left(\mathcal{U} ; \mathcal{A}^{q}\right) \rightarrow C^{p}\left(\mathcal{U} ; \mathcal{A}^{q+1}\right)
$$

la différentielle induite par celle du complexe différentiel $d: \mathcal{A}^{q} \rightarrow \mathcal{A}^{q+1}$. On a $\delta_{2}^{2}=\delta_{1}^{2}=$ 0 et $\delta_{1} \delta_{2}=\delta_{2} \delta_{1}$. Notons

$$
C^{p, q}=\underset{\mathcal{U}}{\lim } C^{p}\left(\mathcal{U} ; \mathcal{A}^{q}\right)
$$


et $\delta_{1}: C^{p, q} \rightarrow C^{p+1, q}, \delta_{2}: C^{p, q} \rightarrow C^{p, q+1}$ les différentielles induites. Le complexe total est noté

$$
K^{\imath}=\oplus_{p+q=i} C^{p, q} \quad d=\delta_{1}+(-1)^{p} \delta_{2}
$$

DÉfinition. L'hypercohomologie du complexe de faisceaux $\mathcal{A}^{\bullet}$ est la cohomologie du complexe simple $\left(K^{\bullet}, d\right)$. On note

$$
\mathbb{I H}^{\imath}\left(X ; \mathcal{A}^{\bullet}\right)=H^{\imath}\left(K^{\bullet}\right)
$$

Si $\mathcal{A}^{\bullet}$ est un complexe non nul seulement en degré $k$ (concentré en degré $k$ ), on a :

$$
\mathrm{IH}^{2}\left(X ; \mathcal{A}^{\bullet}\right)=H^{3-k}\left(X ; \mathcal{A}^{k}\right)
$$

La théorie (classique) des suites spectrales nous montre que si les faisceaux $\mathcal{A}^{q}$ sont acycliques, i.e. si on a pour tout $q$

$$
H^{p}\left(X ; \mathcal{A}^{q}\right)=0 \quad \forall p>0
$$

alors $\mathbb{I H}^{2}\left(X ; \mathcal{A}^{\bullet}\right)$ est le $i$-ème groupe de cohomologie du complexe $\Gamma\left(X ; \mathcal{A}^{\bullet}\right)$.

Des exemples de complexes de faisceaux acycliques sont donnés par les faisceaux injectifs, flasques et, si $X$ est paracompact, par les faisceaux fins ou mous. (voir [8; II, respectivement 7,$1 ; 3.1 ; 3.7 ; 3.4]$, et [ 8 ; II, Théorème 5.2 .3$])$. On peut donc donner comme définition alternative des groupes d'hypercohomologie, ce qui est souvent donné comme définition :

$$
\mathbb{H}^{\imath}\left(X ; \mathcal{A}^{\bullet}\right)=H^{\imath}\left(\Gamma\left(X ; \mathcal{I}^{\bullet}\right)\right)
$$

où $\mathcal{I}^{\bullet}$ est une résolution injective (par exemple) du complexe $\mathcal{A}^{\bullet}$ (isomorphisme $j^{\prime \prime *}$ de $[8 ;$ II.5.3]).

Remarque. Pour toute famille de supports $\Phi$, i.e. ensemble de fermés de $X$ tel que tout sous-fermé d'un élément de $\Phi$ et toute réunion finie d'éléments de $\Phi$ soient dans $\Phi$, on peut définir l'hypercohomologie à supports dans la famille $\Phi: \mathbb{H}_{\Phi}^{2}\left(X ; \mathcal{A}^{\bullet}\right)$. C'est le cas des familles de supports paracompactifiantes, i.e. telles que tout élément de $\Phi$ est paracompact et tout élément de $\Phi$ admet, dans $X$, un voisinage fermé appartenant à $\Phi$.

On donne quelques cas particuliers de groupes d'hypercohomologie, pour $X$ variété algébrique complexe de dimension (réelle) $2 m$.

a) Considérons le complexe de faisceaux dualisant ${ }^{(*)} \mathcal{D}_{X}^{*}=\mathcal{C}_{-}^{F}$. (si $X$ est lisse, $\mathcal{D}_{X}^{*}$ est le faisceau d'orientation $\mathcal{O}_{X}$ ). On a, en prenant pour $\mathcal{A}^{\bullet}$ le complexe décalé $\widetilde{\mathcal{D}}_{X}^{\bullet}=$ $\mathcal{D}_{X}^{\bullet}[-2 m]=\mathcal{D}_{X}^{\bullet-2 m}=\mathcal{C}_{2 m-\bullet}^{F}=\mathcal{C}^{\bullet}$ :

$$
\mathbb{H}^{\imath}\left(X ; \tilde{\mathcal{D}}_{X}^{\bullet}\right) \cong H^{\imath}\left(\Gamma\left(X ; \mathcal{C}^{\bullet}\right)\right)=H^{\imath}\left(\mathcal{C}_{2 m-\bullet}^{F}(X)\right)=H_{2 m-i}^{F}(X),
$$

et, pour la famile des compacts $c$ :

$$
\mathrm{IH}_{c}^{\imath}\left(X ; \widetilde{\mathcal{D}}_{X}^{\bullet}\right)=H_{2 m-i}(X)
$$

\footnotetext{
(*)En fait, on a un quasi-isomorphisme entre le complexe dualisant $\mathcal{D} \bullet$, dont on trouvera une définition intrinsèque dans $[3 ; \mathrm{V}, 7.1]$, et le complexe $\mathcal{C}_{-\bullet}^{F} ;$ voir $[3 ; \mathrm{V}, 7.2]$.
} 
c'est-à-dire l'homologie ordinaire et plus généralement,

$$
\mathbb{I H}_{\Phi}^{i}\left(X ; \widetilde{\mathcal{D}}_{X}^{\bullet}\right)=H_{2 m-i}^{\Phi}(X)
$$

pour toute famille de supports $\Phi([3 ; \mathrm{V}, 7.1-2-3])$.

b) Si $\mathcal{A}^{\bullet}$ est le faisceau constant $\mathbb{Q}_{X}$ sur $X$, considéré comme complexe concentré en degré 0 .

$$
\mathbb{H}^{2}\left(X ; \mathbf{Q}_{X}\right)=H^{2}(X)
$$

cohomologie de $X$ à supports fermés et

$$
\mathbb{I H}_{\Phi}^{2}\left(X ; \mathbb{Q}_{X}\right)=H_{\Phi}^{2}(X)
$$

cohomologie de $X$ à supports dans une famille $\Phi$.

c) Si $\mathcal{A}^{\bullet}$ est le complexe de faisceaux des chaînes d'intersection, à coefficients rationnels et pour la perversité moitié $\mathbf{m}$, noté $\mathcal{I} \mathcal{C}_{X}^{\bullet}$, il vient :

$$
\mathrm{IH}_{c}^{i}\left(X ; \mathcal{I C} \mathcal{C}_{X}^{\bullet}\right)=I H_{2 m-i}(X)
$$

homologie d'intersection à supports compacts, et plus généralement

$$
\mathbb{I H}_{\Phi}^{\imath}\left(X ; \mathcal{I C} \mathcal{C}_{X}^{\bullet}\right)=I H_{2 m-i}^{\Phi}(X)
$$

pour une famille de supports $\Phi([3 ; \mathrm{II}, 5])$.

\section{Dualité de Poincaré.}

3.1. Sur une variété lisse.

Si $X$ est une variété lisse, on a un isomorphisme de complexes : $\mathbb{Q}_{X} \cong \mathcal{C}_{X}^{\bullet}=\tilde{\mathcal{D}}_{X}^{\bullet}$ d'où les isomorphismes

$$
\mathbb{I H}_{\Phi}^{i}\left(X ; \mathbb{Q}_{X}\right) \cong \mathbb{H}_{\Phi}^{\imath}\left(X ; \tilde{\mathcal{D}}_{X}^{\bullet}\right)
$$

c'est-à-dire

$$
H_{\Phi}^{2}(X) \cong H_{2 m-i}^{\Phi}(X)
$$

En particulier, on a

$$
H^{\imath}(X) \cong H_{2 m-i}^{F}(X) \quad \text { et } \quad H_{c}^{2}(X) \cong H_{2 m-i}(X) .
$$

3.2. Sur une variété algébrique complexe de dimension (réelle) pure $2 m$.

Les identifications $\mathbb{Q}_{W} \cong \mathcal{C}_{W}^{\bullet}$ et $\mathcal{C}_{W}^{\bullet} \cong \tilde{\mathcal{D}}_{W}^{\bullet}$ sur un ouvert dense lisse $W \subset X$ induisent des morphismes $\alpha_{X}: \mathbf{Q}_{X} \rightarrow \mathcal{I C} \mathcal{C}_{X}^{\bullet}$ et $\omega_{X}: \mathcal{I C} \mathcal{C}_{X}^{\bullet} \rightarrow \widetilde{\mathcal{D}}_{X}^{\bullet}$ lesquels fournissent les morphismes classiques (de comparaison)

$$
H_{c}^{\bullet}(X) \stackrel{\alpha_{X}}{\longrightarrow} I H_{2 m-\bullet}(X) \quad \text { et } \quad I H_{2 m-\bullet}(X) \stackrel{\omega_{X}}{\longrightarrow} H_{2 m-\bullet}(X)
$$

Leur composé $\delta_{X}:=\omega_{X} \circ \alpha_{X}: \mathbf{Q}_{X} \rightarrow \widetilde{\mathcal{D}}_{X}^{\bullet}$ induit au niveau global l'homomorphisme de

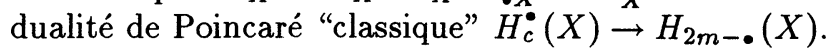

4. Homologie à supports dans une sous-variété fermée. (voir [2])

Soit $X$ fermé de $Y$, on veut expliciter $I H_{\bullet}^{(X)}(Y)$ où $(X)$ désigne la famille des fermés de $Y$ qui sont contenus dans $X$. On a vu que, pour $Y$ de dimension $2 n$, on a

$$
I H_{\bullet}^{\Phi}(Y) \cong H^{2 n-\bullet}\left(\Gamma_{\Phi}\left(Y, \mathcal{A}^{\bullet}\right)\right)
$$


pour une famille de supports $\Phi$ et un complexe $\mathcal{A}^{\bullet} \cong \mathcal{I} \mathcal{C}_{Y}^{\bullet}$ tel que les faisceaux $\mathcal{A}^{q}$ soient $\Gamma_{\Phi}$-acycliques. Or les faisceaux $\mathcal{I} \mathcal{C}_{Y}^{q}$ des chaînes d'intersection sont mous [3; II,5.1] et donc $\Gamma_{\Phi}$-acycliques si la famille $\Phi$ est paracompacifiante, comme c'est le cas pour les familles des fermés et des compacts de $Y$. Mais la famille des fermés d'une sous-variété propre n'est pas paracompactifiante dans la variété ambiante. On ne peut donc pas calculer le groupe d'homologie d'intersection $I H_{\bullet}^{(X)}(Y)$ directement par passage à la cohomologie à partir du complexe $\Gamma_{(X)}\left(Y, \mathcal{I} C_{Y}^{\bullet}\right)$ des sections à support dans le fermé $X$. Nous avons cependant le résultat suivant donnant une l'interprétation géométrique de $I H_{\bullet}^{(X)}(Y)$, même à coefficients dans un anneau arbitraire : phisme

Proposition. (2.3 de [2]) : Soit $X \hookrightarrow Y$ un plongement fermé, on a un isomor-

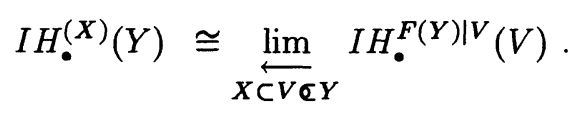

Si de plus le plongement est normalement non-singulier $([9 ; 5.4 .1])$, alors ces deux groupes sont isomorphes à $I H_{\bullet}^{F}(X)$.

\section{Deuxième chapitre}

Les foncteurs image directe et image réciproque.

On désigne par $F a i(X)$ la catégorie abélienne des faisceaux de $\mathbb{Q}$-espaces vectoriels sur $X$. La référence générale de cet exposé est $[3 ; \mathrm{VI}]$.

Pour un morphisme $f: X \rightarrow Y$, on définit deux foncteurs adjoints $f_{*}$ et $f^{*}$ de la façon suivante :

On pose, pour tout ouvert $V$ de $Y$ et tout faisceau $\mathcal{A}$ sur $X$,

$$
f_{*} \mathcal{A}(V)=\Gamma\left(f^{-1}(V) ; \mathcal{A}\right)
$$

et on définit ainsi un foncteur, image directe $f_{*}: F a i(X) \rightarrow F a i(Y)$. Il est exact à gauche et transforme faisceaux injectifs en injectifs. Si $j: X \hookrightarrow Y$ est une inclusion fermée, alors $j_{*} \mathcal{A}=\mathcal{A}^{Y}$ est l'extension de $\mathcal{A}$ par zéro.

Le foncteur image inverse (ou réciproque) $f^{*}: F a i(Y) \rightarrow F a i(X)$ est défini comme suit : soit $\pi: \mathrm{E}(\mathcal{B}) \rightarrow Y$ l'espace étalé associé au faisceau $\mathcal{B}$, par définition $f^{*}(\mathcal{B})$ est le faisceau associé à l'espace étalé $X \underset{Y}{ } \mathrm{E}(\mathcal{B}) \rightarrow X$ obtenu en prenant le produit fibré de $f$ par $\pi$. Pour tout point $x$ de $X$ on a un isomorphisme au niveau des fibres

$$
\left(f^{*} \mathcal{B}\right)_{x}=\mathcal{B}_{f(x)}
$$

Le foncteur $f^{*}$ est exact, il est adjoint du foncteur $f_{*}$. On a

$$
\operatorname{Hom}\left(f^{*} \mathcal{B}, \mathcal{A}\right) \cong \operatorname{Hom}\left(\mathcal{B}, f_{*} \mathcal{A}\right)
$$

Si $j: X \hookrightarrow Y$ est une inclusion alors $j^{*} \mathcal{B}=\left.\mathcal{B}\right|_{X}$ est la restriction de $\mathcal{B}$ à $X$.

En notant $p: X \rightarrow\{\mathrm{pt}\}$ l'application constante, on a $\mathbb{Q}_{X}=p^{*} \mathbb{Q}_{\mathrm{pt}}$, d'où il résulte un isomorphisme canonique $\mathbf{Q}_{X} \cong f^{*} \mathbb{Q}_{Y}$ en utilisant le diagramme commutatif : 


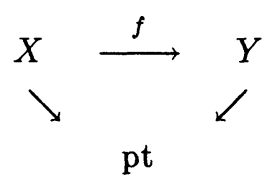

Le foncteurs $f_{1}$.

On définit alors, pour une application continue entre espaces localement compacts, le foncteurs $f_{1}: F a i(X) \rightarrow F a i(Y)$ (image directe à supports propres) comme suit:

Si $V$ est un ouvert de $Y$, on note $\Phi_{V}$ la famille des fermés $E$ de $f^{-1}(V)$ tels que l'application $\left.f\right|_{E}: E \rightarrow V$ soit propre. On définit alors un faisceau $f: \mathcal{A}$ par

$$
f_{1} \mathcal{A}(V)=\Gamma_{\Phi_{V}}\left(f^{-1}(V) ; \mathcal{A}\right) \text {. }
$$

On obtient ainsi un faisceau sur $Y$. Le foncteur $f_{1}$ est exact à gauche et on a un morphisme canonique de foncteurs $0 \longrightarrow f_{1} \longrightarrow f_{*}$ qui, pour une application propre $f: X \rightarrow Y$ est un isomorphisme. Si $Y$ est un point, alors $f_{!} \mathcal{A}=\Gamma_{c}(X ; \mathcal{A})$, où $c$ désigne la famille des compacts de $X$. Si $j: X \hookrightarrow Y$ est une inclusion ouverte ou fermée, alors $j ! \mathcal{A}=\mathcal{A}^{Y}$ et le foncteur $j_{\text {! }}$ est exact. Enfin, le foncteur $f_{1}$ est exact sur la catégorie des faisceaux injectifs sur $X$.

\section{Le foncteurs $f^{!}$.}

Contrairement aux autres foncteurs, ce dernier ne provient pas d'un foncteur au niveau des faisceaux, il n'est défini que dans la catégorie dérivée. On définit d'abord la catégorie $K(X)$ dont les objets sont les complexes de faisceaux de $\mathbb{Q}$-espaces vectoriels sur l'espace $X$ et dont les morphismes sont les classes d'homotopie de morphismes de complexes. $K^{+}(X)$ désigne la sous-catégorie des complexes bornés inférieurement.

La catégorie dérivée $D(X)([3 ; \mathrm{V}, 5.12])$ a les mêmes objets que $K(X)$ mais un morphisme (dans $D(X))$ de $\mathcal{A}^{\bullet}$ dans $\mathcal{B}^{\bullet}$ est une classe d'équivalence de diagrammes de morphismes dans $K(X)$ :

$$
\mathcal{A}^{\bullet} \cong \mathcal{C}^{\bullet} \longrightarrow \mathcal{B}^{\bullet}
$$

où $\cong$ signifie un quasi-isomorphisme. Ce diagramme est équivalent à

$$
\mathcal{A}^{\bullet} \cong \mathcal{C}^{\prime \bullet} \longrightarrow \mathcal{B}^{\bullet}
$$

si il existe $\mathcal{A}^{\bullet} \cong \mathcal{D}^{\bullet} \rightarrow \mathcal{B}^{\bullet}$ et des morphismes $\mathcal{C}^{\bullet} \leftarrow \mathcal{D}^{\bullet} \rightarrow \mathcal{C}^{\bullet}$ tels que l'on ait un diagramme commutatif dans $K(X)$ (i.e. commutatif à homotopie près) :

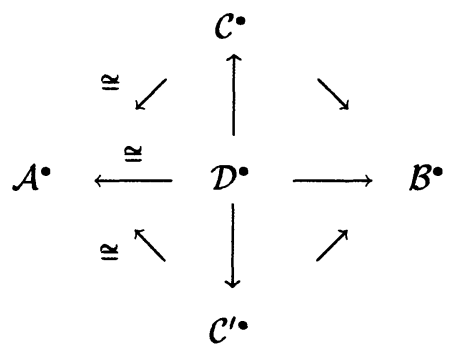

Deux morphismes de $D(X)$ se composent de la façon suivante : étant donné un dia- 
gramme

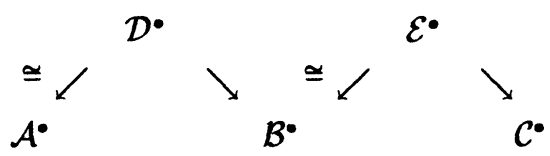

on peut le compléter de manière à obtenir un diagramme commutatif à homotopie près :

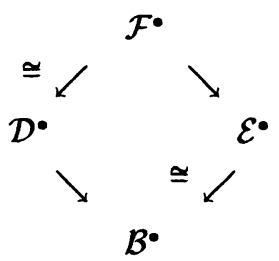

alors $\mathcal{A}^{\bullet} \cong \mathcal{F}^{\bullet} \longrightarrow \mathcal{C}^{\bullet}$ est la composition des morphismes donnés.

On définit de même la catégorie dérivée $D^{+}(X)$ des complexes bornés inférieurement.

Etant données une application continue d'espaces localement compacts, et une "bonne" résolution du faisceau constant $\mathbb{Q}$ sur $X: 0 \rightarrow \mathbb{Q} \rightarrow \mathcal{R}^{\bullet}$, on définit d'abord un foncteur

$$
f_{\mathcal{R}^{\bullet}}^{!}: K^{+}(Y) \longrightarrow K^{+}(X)
$$

par

$$
f_{\mathcal{R}}^{!} \cdot\left(\mathcal{G}^{\bullet}\right)(U)=H_{o m}^{\bullet}\left(f_{!}\left(\mathcal{R}_{U}^{\bullet}\right) ; \mathcal{G}_{U}^{\bullet}\right)
$$

La correspondance $\mathcal{G}^{\bullet} \mapsto f_{\mathcal{R}^{\bullet}}^{!}\left(\mathcal{G}^{\bullet}\right)$ définit un foncteur $f_{\mathcal{R}^{\bullet}}^{!}: K^{+}(Y) \rightarrow K^{+}(X)$. Si $\mathcal{R}^{\bullet}$ et $\mathcal{S}^{\bullet}$ sont deux "bonnes" résolutions du faisceau $\mathbb{Q}$, alors les complexes de faisceaux $f_{\mathcal{R}^{\bullet}}^{\prime}\left(\mathcal{G}^{\bullet}\right)$ et $f_{\mathcal{S}}^{\prime} \bullet\left(\mathcal{G}^{\bullet}\right)$ sont quasi-isomorphes. On en déduit que le foncteur

$$
f^{!}: D^{+}(Y) \longrightarrow D^{+}(X)
$$

induit sur les catégories dérivées par le foncteur $f_{\mathcal{R}}^{!}$• est indépendant, à isomorphisme près, du choix de la résolution $\mathcal{R}^{\bullet}$.

Remarque : La dualité entre les foncteurs $f_{1}$ et $f^{\prime}$ s'exprime dans la catégorie dérivée, au moyen de foncteurs dérivés, de la façon suivante (voir $[3 ; \mathrm{V}, 7.17])$ :

$$
R H o m^{\bullet}\left(R f_{!} \mathcal{A}^{\bullet}, \mathcal{B}^{\bullet}\right)=R f_{*} R H o m^{\bullet}\left(\mathcal{A}^{\bullet}, f^{!} \mathcal{B}^{\bullet}\right)
$$

Si $j: X \hookrightarrow Y$ est une inclusion ouverte, on a $j^{!}=j^{*}$; si c'est une inclusion fermée, alors on a

$$
j^{!}\left(\mathcal{G}^{\bullet}\right)(U)=\Gamma_{(X)}\left(V ; \mathcal{G}^{\bullet}\right)
$$

où $V$ est un ouvert de $Y$ tel que $U=V \cap X$. Si $Y$ est un point et $p: X \rightarrow\{\mathrm{pt}\}$ l'application constante et $\mathcal{B}^{\bullet}=\mathbb{Q}_{\mathrm{pt}}$, il vient $([3 ; \mathrm{V}, 7.18])$

$$
\mathcal{D}_{X}^{\bullet} \cong p^{!} \mathbb{Q}_{\mathrm{pt}}
$$

d'où pour tout $f: X \rightarrow Y$ un isomorphisme canonique $\mathcal{D}_{X}^{\bullet} \cong f^{\prime} \mathcal{D}_{Y}^{\bullet}$.

\section{Morphismes associés en homologie et cohomologie.}

Soient $\mathcal{A}^{\bullet}$ et $\mathcal{B}^{\bullet}$ des complexes bornés de faisceaux de $\mathbf{Q}$-espaces vectoriels, et $\Phi$ et $\Psi$ des familles de supports sur les variétés $X$ et $Y$ respectivement. Notons $f^{-1}(\Psi)$ la famille des fermés de $X$ qui s'écrivent $f^{-1}(E)$ avec $E \in \Psi$ et notons $\Lambda_{f}$ la famille 
des fermés $F$ de $X$ qui sont propres sur $Y$ par rapport à $f$, i.e. tels que la restriction $\left.f\right|_{F}: F \rightarrow Y$ soit une application propre. Alors on a le résultat suivant :

Proposition. (Lemme du $§ 4$ de [2]) : a) Si la famille $f^{-1}(\Psi)$ est contenue dans $\Phi$, alors tout morphisme de complexes de faisceaux $f^{*} \mathcal{B}^{\bullet} \rightarrow \mathcal{A}^{\bullet}$ sur $X$ induit un homomorphisme naturel en hypercohomologie

$$
\mathbb{I H}_{\mathbf{\Psi}}^{\bullet}\left(Y ; \mathcal{B}^{\bullet}\right) \longrightarrow \mathbb{H}_{\Phi}^{\bullet}\left(X ; \mathcal{A}^{\bullet}\right)
$$

b) Si la famille $\Phi$ est contenue dans $\Lambda_{f} \cap f^{-1}(\Psi)$, alors tout morphisme de complexes de faisceaux $\mathcal{A}^{\bullet} \rightarrow f^{!} \mathcal{B}^{\bullet}$ sur $X$ induit un homomorphisme naturel en hypercohomologie

$$
\mathrm{IH}_{\Phi}^{\bullet}\left(X, \mathcal{A}^{\bullet}\right) \longrightarrow \mathrm{IH}_{\Psi}^{\bullet}\left(Y, \mathcal{B}^{\bullet}\right) \text {. }
$$

Démonstratıon. On obtient le premier résultat en composant les homomorphismes naturels évidents

$$
\mathbb{H}_{\Psi}^{\bullet}\left(Y ; \mathcal{B}^{\bullet}\right) \longrightarrow \mathbb{H}_{f^{-1}(\Psi)}^{\bullet}\left(X ; f^{*} \mathcal{B}^{\bullet}\right) \longrightarrow \mathbb{H}_{f^{-1}(\Psi)}^{\bullet}\left(X ; \mathcal{A}^{\bullet}\right) \longrightarrow \mathbb{H}_{\Phi}^{\bullet}\left(X ; \mathcal{A}^{\bullet}\right)
$$

Pour le deuxième énoncé, on compose les homomorphismes canoniques

$$
\mathbb{I}_{\Phi}^{\bullet}\left(X, \mathcal{A}^{\bullet}\right) \longrightarrow \mathbb{H}_{\Lambda_{f} \cap^{-1}(\Psi)}\left(X, \mathcal{A}^{\bullet}\right) \stackrel{\cong}{\longrightarrow} \mathbb{H}_{\Psi}^{\bullet}\left(Y, f_{!} \mathcal{A}^{\bullet}\right) \longrightarrow \mathbb{H}_{\Psi}^{\bullet}\left(Y, \mathcal{B}^{\bullet}\right) .
$$

L'existence de l'isomorphisme du milieu est montrée par une généralisation de la formule $[3 ; \mathrm{V}, 7.11(2)]$; pour la dernière flèche, on applique le foncteur $\mathbb{H}_{\Psi}^{\bullet}(Y,-)$ au morphisme $f: \mathcal{A}^{\bullet} \rightarrow \mathcal{B}^{\bullet}$ sur $Y$ obtenu par adjonction du morphisme donné $\mathcal{A}^{\bullet} \rightarrow f^{!} \mathcal{B}^{\bullet}$ d'après la dualité de Verdier (dans la catégorie dérivée [ $3 ; \mathrm{V}, 7.17]$ ).

Notons respectivement $c(X)$ et $F(X)$ les familles des compacts et des fermés de $X$, on remarque que $f: X \rightarrow Y$ est propre si et seulement si $f^{-1}(c(Y))=c(X)$ ou $F(X) \subset \Lambda_{f}=\Lambda_{f} \cap f^{-1}(F(Y))$. Notons $\operatorname{dim} X=2 m, \operatorname{dim} Y=2 n$ et $2 s=2(n-m)$.

\section{Corollaire.}

1) L'isomorphisme $f^{*}\left(\mathbb{Q}_{Y}\right) \stackrel{\cong}{\longrightarrow} \mathbb{Q}_{X}$ induit $\mathbb{H}_{\Psi}^{\bullet}\left(Y, \mathbf{Q}_{Y}\right) \longrightarrow \mathbb{H}_{\Phi}^{\bullet}\left(X, \mathbf{Q}_{X}\right)$ donc pour $\Phi$ et $\Psi$ les familles des fermés de $X$ et $Y, H^{\bullet}(Y) \stackrel{f^{*}}{\longrightarrow} H^{\bullet}(X)$ et, si $f$ est propre, pour les familles de compacts, $H_{c}^{\bullet}(Y) \stackrel{f^{*}}{\longrightarrow} H_{c}^{\bullet}(X)$.

2) Tout morphisme $\mu: f^{*}\left(\mathcal{I C} \mathcal{C}_{Y}^{\bullet}\right) \longrightarrow \mathcal{I C} \mathcal{C}_{X}^{\bullet}$ induit $\mathbb{H}_{\Psi}^{\bullet}\left(Y, \mathcal{I C} \mathcal{C}_{Y}^{\bullet}\right) \longrightarrow \mathbb{H}_{\Phi}^{\bullet}\left(X, \mathcal{I C} \mathcal{C}_{X}^{\bullet}\right)$ donc pour $\Phi$ et $\Psi$ les familles des fermés de $X$ et $Y, I H_{2 n-\bullet}^{F}(Y) \stackrel{\mu}{\longrightarrow} I H_{2 m-\bullet}^{F}(X)$ et, si $f$ est propre, pour les familles de compacts, $I H_{2 n-\bullet}(Y) \stackrel{\mu}{\longrightarrow} I H_{2 m-\bullet}(X)$.

3) L'isomorphisme $\tilde{\mathcal{D}}_{X}^{\bullet} \stackrel{\cong}{\longrightarrow} f^{\prime}\left(\widetilde{\mathcal{D}}_{Y}^{*}\right)[2 s]$ induit $\mathbb{H}_{\Phi}^{\bullet}\left(X, \tilde{\mathcal{D}}_{X}^{*}\right) \longrightarrow \mathbb{H}_{\Psi}^{\bullet}\left(Y, \tilde{\mathcal{D}}_{Y}^{*}[2 s]\right)$ donc pour $\Phi$ et $\Psi$ les familles des compacts de $X$ et $Y, H_{2 m-\bullet}(X) \stackrel{f_{*}}{\longrightarrow} H_{2 m-\bullet}(Y)$ et, si $f$ est propre, pour les familles de fermés, $H_{2 m-\bullet}^{F}(X) \stackrel{f_{*}}{\longrightarrow} H_{2 m-\bullet}^{F}(Y)$.

4) Tout morphisme $\nu: \mathcal{I C} \mathcal{C}_{X}^{\bullet} \longrightarrow f^{!}\left(\mathcal{I C} \mathcal{C}_{Y}^{\bullet}\right)[2 s]$ induit $\mathbb{H}_{\Phi}^{\bullet}\left(X, \mathcal{I C} \mathcal{C}_{X}^{\bullet}\right) \longrightarrow \mathbb{H}_{\Psi}^{\bullet}\left(Y, \mathcal{I C} C_{Y}^{\bullet}[2 s]\right)$ donc pour $\Phi$ et $\Psi$ les familles des compacts de $X$ et $Y, I H_{2 m-\bullet}(X) \stackrel{\nu}{\longrightarrow} I H_{2 m-\bullet}(Y)$ et, si $f$ est propre, pour les familles de fermés, $I H_{2 m-\bullet}^{F}(X) \stackrel{\nu}{\longrightarrow} I H_{2 m-\bullet}^{F}(Y)$. 


\section{Troisième chapitre}

La référence générale de cet exposé est [2], les parenthèses renvoient à cette référence.

\section{Motivation.}

A titre de motivation, on développe, sans démonstration, une idée de construction géométrique de morphismes associés en homologie d'intersection en supposant l'existence d'un relèvement de la classe du graphe de $f$ en homologie d'intersection à coefficients rationnels et à supports convenables.

Soit $f: X \longrightarrow Y$ un morphisme de variétés algébriques complexes, connexes et de dimensions pures respectives $m$ et $n$, nous notons $\Gamma_{f}$ le graphe de $f$, cycle algébrique de $X \times Y$. La formule ensembliste $f(A)=\operatorname{pr}_{Y}\left((A \times Y) \cap \Gamma_{f}\right)$, pour un sous-ensemble $A$ de $X$, suggère de considérer l'application suivante : Etant donné un cycle d'intersection $\gamma$ dans $X \times Y$, qui relève $\Gamma_{f}$, nous faisons correspondre à tout cycle d'intersection $\xi$ de $X$ le cycle d'intersection $\operatorname{pr}_{Y}((\xi \times[Y]) \cap \gamma)$ de $Y$.

Afin de préciser cette idée, nous introduisons, pour deux perversités $\mathbf{p}$ et $\mathbf{q}$ données, l'homologie d'intersection "produit" (voir $[3 ; \mathrm{V}, 6.2(3)]$ pour la définition de $\stackrel{L}{\otimes}$ )

$$
I_{(\mathbf{p}, \mathbf{q})} H_{\bullet}(X \times Y):=\mathbb{H}_{c}^{2 m+2 n-\bullet}\left(X \times Y, p_{1}^{*} \mathcal{I}_{\mathbf{p}} \mathcal{C}_{X}^{\bullet} \stackrel{L}{\otimes} p_{2}^{*} \mathcal{I}_{\mathbf{q}} \mathcal{C}_{Y}^{\bullet}\right)
$$

par rapport à la "biperversité" $(\mathbf{p}, \mathbf{q})$ et avec pour $p_{i}$ les projections canoniques. On a toujours l'isomorphisme

$$
I_{(\mathbf{p}, \mathbf{q})} H_{\bullet}(X \times Y) \cong I_{\mathbf{p}} H_{\bullet}(X) \otimes I_{\mathbf{q}} H_{\bullet}(Y)
$$

valable pour des perversités arbitraires (cf. [3; V, 10.19]). Le produit d'intersection usuel induit un produit d'intersection

$$
I_{\left(\mathbf{p}, \mathbf{p}^{\prime}\right)} H_{i}(X \times Y) \times I_{\left(\mathbf{q}, \mathbf{q}^{\prime}\right)} H_{j}(X \times Y) \stackrel{\cap}{\longrightarrow} I_{\left(\mathbf{r}, \mathbf{r}^{\prime}\right)} H_{\imath+\jmath-2(m+n)}(X \times Y)
$$

pour $\mathbf{r} \geq \mathbf{p}+\mathbf{q}$ et $\mathbf{r}^{\prime} \geq \mathbf{p}^{\prime}+\mathbf{q}^{\prime}$.

Pour l'homologie d'intersection du produit, on a un isomorphisme "de Künneth" :

$$
I_{\mathbf{p}} H_{\bullet}(X \times Y) \cong I_{(\mathbf{p}, \mathbf{p})} H_{\bullet}(X \times Y)
$$

valable pour toutes les perversités $\mathbf{p}$ satisfaisant à la condition

$$
\mathbf{p}(2 k)+\mathbf{p}(2 l) \leq \mathbf{p}(2 k+2 l) \leq \mathbf{p}(2 k)+\mathbf{p}(2 l)+2 .
$$

et provenant, pour ces perversités, d'une autre formule de Künneth

$$
\mathcal{I}_{\mathbf{p}} \mathcal{C}_{\boldsymbol{X} \times Y}^{\bullet} \cong p_{1}^{*} \mathcal{I}_{\mathbf{p}} \mathcal{C}_{X}^{\bullet} \stackrel{L}{\otimes} p_{2}^{*} \mathcal{I}_{\mathbf{q}} \mathcal{C}_{Y}^{\bullet}
$$

au niveau des complexes de faisceaux (voir [7]). La condition précédente est vérifiée dans les cas particuliers les plus importants $\mathbf{p}=\mathbf{o}, \mathbf{m}$ (cf. $[9 ; 6.3]$ ) et $\mathbf{t}$. Supposons que les variétés $X$ et $Y$ soient compactes et faisons l'hypothèse que la classe d'homologie $\left[\Gamma_{f}\right] \epsilon$ $H_{2 m}(X \times Y)$ se relève en une classe $\gamma$ dans $I_{\mathbf{m}} H_{2 m}(X \times Y) \cong I_{(\mathbf{m}, \mathbf{m})} H_{2 m}(X \times Y)$. Comme la classe fondamentale $[Y]$ appartient d'une manière naturelle à $I_{\mathbf{O}} H_{2 n}(Y)$, on peut définir un homomorphisme $\nu_{\gamma}: I H_{\bullet}(X) \rightarrow I H_{\bullet}(Y)$ par la composition :

$$
\begin{array}{ccccc}
I_{\mathbf{m}} H_{\bullet}(X) & \rightarrow I_{(\mathbf{m}, \mathbf{o})} H_{\bullet+2 n}(X \times Y) & \rightarrow I_{(\mathbf{t}, \mathbf{m})} H_{\bullet}(X \times Y) & \rightarrow & I_{\mathbf{m}} H_{\bullet}(Y) \\
\xi & \mapsto & \xi \times[Y] & \mapsto \quad(\xi \times[Y]) \cap \gamma & \mapsto q_{\bullet}((\xi \times[Y]) \cap \gamma)
\end{array}
$$


où $q$. désigne la projection canonique

$$
I_{(\mathbf{t}, \mathbf{m})} H_{\bullet}(X \times Y) \cong I_{\mathbf{t}} H_{\bullet}(X) \otimes I_{\mathbf{m}} H_{\bullet}(Y) \rightarrow I_{\mathbf{t}} H_{0}(X) \otimes I_{\mathbf{m}} H_{\bullet}(Y) \cong I_{\mathbf{m}} H_{\bullet}(Y)
$$

en utilisant l'isomorphisme $I_{\mathbf{t}} H_{0}(X) \cong H_{0}(X) \cong \mathbb{Q}$ dû au fait que $X$ est connexe. Un tel morphisme $\nu_{\gamma}$ est un bon candidat pour un morphisme associé $\nu_{f}$. Pour passer au cas où les variétés ne sont pas nécessairement compactes, on a en fait besoin d'un relèvement de la classe $\left[\Gamma_{f}\right]$, vue comme élément de $H_{2 m}^{\left(\Gamma_{f}\right)}(X \times Y)$, homologie à supports dans la famille des fermés contenus dans $\Gamma_{f}$, admet un relèvement $\gamma$ dans $I H_{2 m}^{\left(\Gamma_{f}\right)}(X \times Y)$.

\section{Le théorème d'existence local.}

Dans toute la suite, nous considérerons tous les complexes et groupes d'homologie d'intersection par rapport à la perversité moitié. Le théorème d'existence de morphismes associés s'exprime dans le langage des faisceaux :

THÉORÈME PRINCIPAL (2.1). Sort $f: X \rightarrow Y$ un morphisme entre variétés algébriques complexes de dimensions pures $m$ et $n$ respectıvement. Alors, il existe des morphismes contravariants $\mu^{f}: f^{*}\left(\mathcal{I} \mathcal{C}_{Y}^{\bullet}\right) \rightarrow \mathcal{I} \mathcal{C}_{X}^{\bullet}$ et covariants $\nu_{f}: \mathcal{I C} \mathcal{C}_{X}^{\bullet} \rightarrow f^{!}\left(\mathcal{I C} \mathcal{C}_{Y}^{\bullet}\right)[2 s]$ (où nous posons $s:=n-m$ ) de complexes de fassceaux de chaînes d'intersection à coefficıents rationnels quı sont associés à $f$, c'est-à-dire qu'ils sont compatibles avec les morphismes de comparaison $\alpha$ et $\omega$ au sens de la commutatıvité des diagrammes suivants :

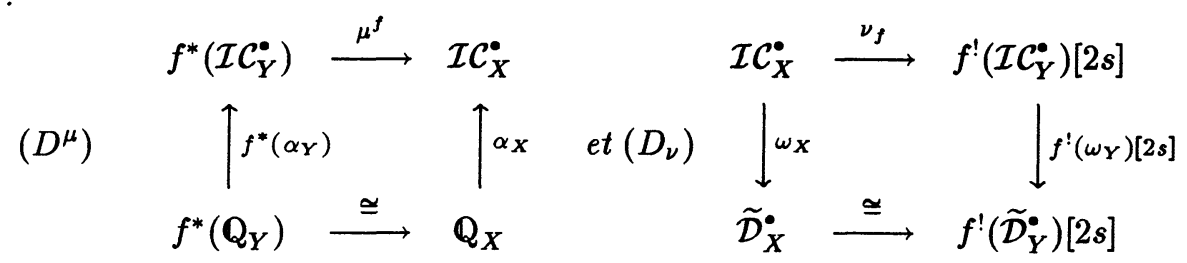

Les morphısmes contravariants $\mu^{f}$ et covariants $\nu_{f}$ sont en correspondance bıunivoque, par dualité de Poincaré-Verdier.

REMARQUE SUR L'UNICITÉ. Les morphismes associés $\mu^{f}$ et $\nu_{f}$ sont determinés de façon unique par $f$ dans certains cas particuliers, par exemple :

a) si $Y$ est lisse (alors $\mu^{f}$ est déterminé par $\alpha_{X}$ puisque $\alpha_{Y}$, et donc $f^{*}\left(\alpha_{Y}\right)$, sont des isomorphismes),

b) si $f$ est un morphisme dominant équidimensionnel,

c) si $f$ est le plongement d'une sous-variété fermée $X$ de codimension 1 dans $Y$ telle que $Y$ soit localement analytiquement irréductible le long de $X$,

d) si $f$ est une application "petite" dans le sens de $[9 ; 6.2]$.

Dans les cas a), b) et d), le morphisme $\mu^{f}$ existe même à coefficients entiers.

\section{Le théorème d'existence global :}

La première conséquence du Théorème principal est l'existence d'homomorphismes globaux associés à $f$ dont nous énonçons les cas particuliers les plus intéressants : 
THÉORÈME (2.2). Sous les hypothèses du Théorème 2.1, nous avons les résultats survants :

1) Il existe des homomorphismes contravariants $\mu^{f}=\mu_{F}^{f}: I H_{2 n-\bullet}^{F}(Y) \rightarrow I H_{2 m-\bullet}^{F}(X)$ à supports fermés, et covariants $\nu_{f}=\nu_{f}^{c}: I H_{\bullet}(X) \rightarrow I H_{\bullet}(Y)$ à supports compacts quı sont associés au morphisme $f$, i.e. qui rendent commutatifs les diagrammes survants :

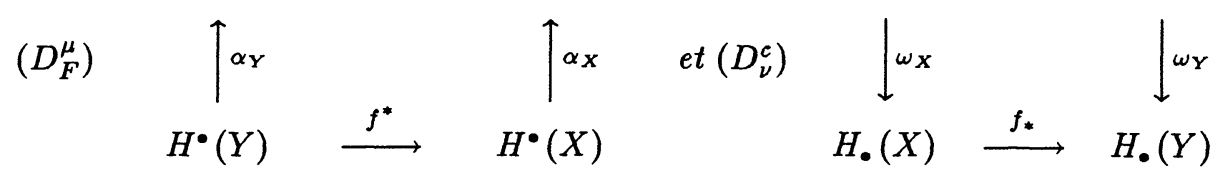

2) Si l'application $f: X \rightarrow Y$ est propre, alors il existe des homomorphismes associés contravariants à supports compacts $\mu^{f}=\mu_{c}^{f}: I H_{2 n-\bullet}(Y) \rightarrow I H_{2 m-\bullet}(X)$ et covariants à supports fermés $\nu_{f}=\nu_{f}^{F}: I H_{\bullet}^{F}(X) \rightarrow I H_{\bullet}^{F}(Y)$ qui rendent commutatifs les diagrammes analogues:

$\left(D_{c}^{\mu}\right)$

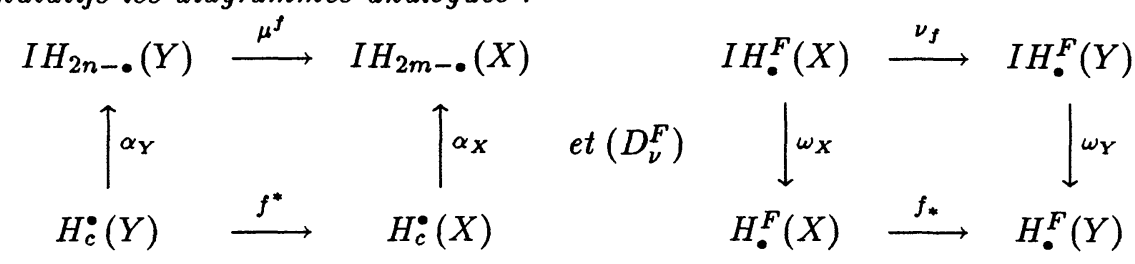

\section{Le théorème de relèvement :}

La deuxième conséquence du théorème principal concerne le relèvement des cycles algébriques en homologie d'intersection. Pour l'énoncer, nous utilisons la notion d'homologie d'intersection $I H_{\bullet}^{(X)}(Y)$ de $Y$ à supports dans une sous-variété fermée $X$ de $Y$, c'est-à-dire l'homologie d'intersection relative $I H_{\bullet}^{F}(Y, Y \backslash X)$ (voir ci-dessus) :

THÉORÈme (2.3). Si $X$ est une sous-variété fermée de $Y$, de dimension pure $m$, alors la classe d'homologıe $[X]$ (à coefficıents ratıonnels) est dans l'image de l'homomorphisme de comparaison:

$$
I H_{2 m}^{(X)}(Y) \longrightarrow H_{2 m}^{(X)}(Y)
$$

En particulier, la classe d'homologie dans $H_{2 m}^{F}(Y)$ de tout cycle algébrique $X$ dans $Y$, contient un cycle d'intersection $\xi$ arbitrairement proche de $X$, i.e. étant donné un voisinage $V$ de $X$ dans $Y$, on peut supposer $|\xi| \subset V$.

DÉmonstration DU ThÉorème 2.3. Notons d'abord que la classe d'homologie $[X]$ d'un cycle algébrique $X \subset Y$ peut être interprétée comme élément de $H_{2 m}^{(X)}(Y) \cong$ $H_{2 m}^{F}(X)$. L'existence d'un relèvement de cette classe en homologie d'intersection résulte immédiatement du diagramme commutatif 


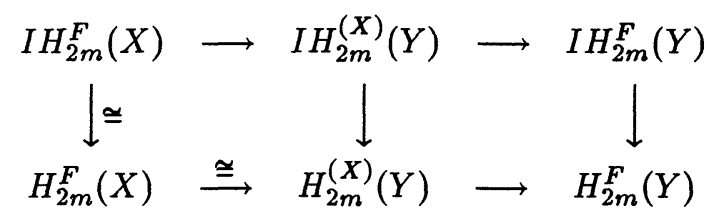

Puisque les classes de Chern (au sens de Schwartz [11] et MacPherson [10]) d'une variété algébrique, en homologie, sont représentables par des cycles algébriques, le Théorème de relèvement nous permet de répondre positivement à la conjecture bien connue (voir $[3 ; \mathrm{IX}, \mathrm{H}]$ et $[1 ; \S 3]$ ) concernant le relèvement de ces classes :

CoRollaire. Les classes de Chern-Schwartz-MacPherson d'une variété algébrique, en homologıe, se relèvent en homologıe d'intersection, pour la perversité moıtıé et à coefficients ratıonnels.

Malheureusement il n'y a pas, en général, de relèvement canonique comme le montre l'exemple donné dans [4]. D'autre part, les résultats précédents, valables pour la perversité moitié (et les perversités supérieures) ne permettent pas (directement) de multiplier plus de deux classes d'homologie, ce qui est un obstacle à la définition de nombres caractéristiques généraux pour les variétés algébriques complexes singulières.

\section{Le théorème de classification :}

Nous avons déjà remarqué que les morphismes $\mu^{f}$ ne sont pas uniquement déterminés par le morphisme $f$. Le résultat suivant permet de déterminer le degré de liberté de cette ambigüité. En même temps, il précise le raisonnement géométrique de la motivation et complète le théorème principal :

THÉORÈME (2.4). Il y a une correspondance bıunıvoque entre les morphismes $\mu^{f}$, resp. $\nu_{f}$, rendant commutatif le diagramme $\left(D^{\mu}\right)$, resp. $\left(D_{\nu}\right)$, du théorème princıpal, et les classes $\gamma \in I H_{2 m}^{\left(\Gamma_{f}\right)}(X \times Y)$ quı relèvent la classe d'homologıe $\left[\Gamma_{f}\right] \in H_{2 m}^{\left(\Gamma_{f}\right)}(X \times Y)$.

De manière plus précise :

Remarque. Les trois énoncés suivants sont équivalents (même à coefficients dans un corps quelconque) :

1) Tout cycle algébrique $C$ d'une variété algébrique $Z$ est homologue à un cycle d'intersection $\xi$ arbitrairement proche de $C$ dans $Z$.

2) Pour tout morphisme $f: X \rightarrow Y$ entre variétés algébriques, il existe un homomorphisme associé $\mu^{f}: f^{*}\left(\mathcal{I C} \mathcal{C}_{Y}^{*}\right) \rightarrow \mathcal{I C} \mathcal{C}_{X}^{*}$.

3) Pour tout morphisme $f: X \rightarrow Y$ entre variétés algébriques, il existe un homomorphisme associé $\nu_{f}: \mathcal{I} \mathcal{C}_{X}^{\bullet} \rightarrow f^{!}\left(\mathcal{I C} \mathcal{C}_{Y}^{\bullet}\right)[2 s]$

\section{REFERENCE}

[1] Gottfried Barthel, Jean-Paul Brasselet et Karl-Heinz Fieseler : Classes de Chern des variétés 
toriques singulières. C.R. Acad. Sci. Paris, t. 315, Série I, p. 187-192, 1992.

[2] Gottfried Barthel, Jean-Paul Brasselet, Karl-Heinz Fieseler, Ofer Gabber et Ludger Kaup : Relèvement des cycles algébriques et homomorphismes associés en homologie d'intersection, Annals of Maths 139 (1994), 1-33.

[3] Armand Borel et al. : Intersection Cohomology. Progress in Math. vol. 50, Birkhäuser 1984, Boston etc. (Borel-Seminar).

[ 4 ] Jean-Paul Brasselet et Gerardo Gonzalez-Sprinberg : Sur l'homologie d'intersection et les classes de Chern des variétés singulières. Travaux en cours $n^{\circ} 23$, Hermann 1987, 5-11.

[ 5 ] Séminaire Cartan, 1948/49, Exposé 5 de Dixmier.

[6] Séminaire Cartan, 1950/51 Exposé 15 de Cartan.

[ 7 ] Dan Cohen, Mark Goresky and Lizhen Ji : On the Künneth Formula for Intersection Cohomology. Preprint Northeastern University, Dec. 1989.

[8] Roger Godement : Théorie des faisceaux. Actualités scientifiques et industrielles. Hermann, Paris, 1964.

[ 9 ] Mark Goresky and Robert MacPherson :, Intersection homology theory II. Inv. Math. 71 (1983), 77-129.

[10] Robert MacPherson : Chern Classes for Singular Algebraic Varieties. Annals of Math. 100 (1974), 423-432.

[11] Marie-Hélène Schwartz : Classes caractéristiques définies par une stratification d'une variété analytique complexe. C.R. Acad. Sci. Paris, t. 260, Série I, p. 3262-3264 et 3535-3537, 1965.

CIRM LUMINY

CASE 916

13288 Marseille Cedex 9

FRANCE 